\title{
SIMPLIFIED PROOFS OF THREE THEOREMS ON THE KINEMATICS OF AXISYMMETRIC DEFORMATION OF SHELLS OF REVOLUTION
}

\author{
BY \\ J. G. SIMMONDS \\ University of Virginia, Charlottesville
}

Introduction. In [1] Naghdi and Vongsarnpigoon establish the following three theorems for shells of revolution suffering torsionless, axisymmetric deformation.

THEOREM 1. The surface components of the strain ... determine the coefficients of the second fundamental form ... of the deformed configuration.... Furthermore, the deformation of the shell-like body (including the local rotation...) is then determined.

THEOREM 2. For a deforming shell which is nonshallow in the reference state..., if the components of strain ... and its first- and higher-order gradients are all infinitesimal of $O(\varepsilon)$, then the [relative] deformation is either infinitesimal or one with small strain accompanied by large rotation. The latter necessarily involves an inversion of the shell.

Theorem 3. For a shallow shell of revolution, if the components of strain ... and the first- and higher-order gradients are infinitesimal of $O(\varepsilon)$, then the [relative] deformation is at most one with small strain of $O(\varepsilon)$ accompanied by moderate rotation of $O\left(\varepsilon^{1 / 2}\right)$.

Naghdi and Vongsarnpigoon prove these using a Cosserat (director) model of a shell, tensor analysis, the polar decomposition theorem, and results from general surface theory. Note that these three theorems concern extensional (membrane) strains only and thus hold regardless of what model-e.g., Kirchhoff-Love or director-is evoked to compute deformations away from the shell reference surface.

My aim is to show that these theorems follow almost immediately from a simple compatibility condition given thirty-five years earlier by Reissner [2, Eq. (20)]. ${ }^{1}$ Moreover, I show that one of the conclusions of Theorem 2 can be tightened and that the last part of Theorem 3 should be modified to read “... then the relative

\footnotetext{
Received January 3, 1992.

1991 Mathematics Subject Classification. Primary 73.

This research was supported by the National Science Foundation under Grant MSS-9102155.

${ }^{1}$ Reissner simplifies his kinematical results by assuming infinitesimal extensional strains and moderate rotations after his Eqs. (15) and (16) of which his Eq. (20) is an exact consequence. Thus this latter equation is valid for any smooth deformation of the reference surface.
}

(C)1994 Brown University 
radial deformation is $O(\varepsilon)$ and the relative axial deformation, modulo a translation, is $O\left(\varepsilon^{1 / 2}\right)$, accompanied by a rotation that is moderate of $O\left(\varepsilon^{1 / 2}\right)$, modulo $2 \pi$." Finally, I show that only the gradient of the hoop strain need be $O(\varepsilon)$.

Analysis. Let the reference surface of a shell of revolution be given in the vector parametric form

$$
\mathscr{R}: \mathbf{r}=r(\sigma) \mathbf{e}_{r}(\theta)+z(\sigma) \mathbf{e}_{z}, \quad \sigma \in[0, L] .
$$

Here, $\sigma$ is arc length along an undeformed meridian of $\mathscr{R}$, and $\mathbf{e}_{r}$ and $\mathbf{e}_{z}$ are the standard orthonormal base vectors in the $(r, z)$-plane of the circular cylindrical coordinate system $(r, \theta, z)$. For simplicity, I assume that $r(\sigma)$ and $z(\sigma)$ and their deformed images, $\bar{r}(\sigma)$ and $\bar{z}(\sigma)$, are continuous with piecewise continuous first and second derivatives on $(0, L)$.

A unit tangent to a meridian of $\mathscr{R}$ is

$$
\begin{aligned}
\mathbf{r},{ }_{\sigma}(\sigma, \theta) & =r^{\prime}(\sigma) \mathbf{e}_{r}(\theta)+z^{\prime}(\sigma) \mathbf{e}_{z} \\
& \equiv \cos \alpha(\sigma) \mathbf{e}_{r}(\theta)+\sin \alpha(\sigma) \mathbf{e}_{z},
\end{aligned}
$$

where a comma followed by a subscript indicates differentiation with respect to that subscript. A unit normal to $\mathscr{R}$ is

$$
\mathbf{b}=-\sin \alpha(\sigma) \mathbf{e}_{r}(\theta)+\cos \alpha(\sigma) \mathbf{e}_{z}
$$

The first and second fundamental forms on $\mathscr{R}$ are, respectively,

$$
d \mathbf{r} \cdot d \mathbf{r}=d \sigma^{2}+r^{2}(\sigma) d \theta^{2}
$$

and

$$
-d \mathbf{r} \cdot d \mathbf{b}=\alpha^{\prime}(\sigma) d \sigma^{2}+r(\sigma) \sin \alpha(\sigma) d \theta^{2} .
$$

Under a static, torsionless, axisymmetric deformation, $\mathscr{R}$ goes into some new surface of revolution

$$
\overline{\mathscr{R}}: \overline{\mathbf{r}}=\bar{r}(\sigma) \mathbf{e}_{r}(\theta)+\bar{z}(\sigma) \mathbf{e}_{z}, \quad \sigma \in[0, L] .
$$

A tangent to a meridian of $\overline{\mathscr{R}}$ is

$$
\begin{aligned}
\overline{\mathbf{r}},{ }_{\sigma}(\sigma, \theta) & =\vec{r}^{\prime}(\sigma) \mathbf{e}_{r}(\theta)+\bar{z}^{\prime}(\sigma) \mathbf{e}_{z} \\
& \equiv \lambda(\sigma)\left[\cos \bar{\alpha}(\sigma) \mathbf{e}_{r}(\theta)+\sin \bar{\alpha}(\sigma) \mathbf{e}_{z}\right],
\end{aligned}
$$

where I assume that $\lambda>0$; a unit normal to $\overline{\mathscr{R}}$ is

$$
\overline{\mathbf{b}}=-\sin \bar{\alpha}(\sigma) \mathbf{e}_{r}(\theta)+\cos \bar{\alpha}(\sigma) \mathbf{e}_{z} .
$$

Thus the first and second fundamental forms on $\overline{\mathscr{R}}$ are

$$
d \overline{\mathbf{r}} \cdot d \overline{\mathbf{r}}=\lambda^{2}(\sigma) d \sigma^{2}+\bar{r}^{2}(\sigma) d \theta^{2}
$$

and

$$
-d \overline{\mathbf{r}} \cdot d \overline{\mathbf{b}}=\bar{\alpha}^{\prime}(\sigma) d \sigma^{2}+\bar{r}(\sigma) \sin \bar{\alpha}(\sigma) d \theta^{2} .
$$

Once the deformed tangent angle $\bar{\alpha}$, the meridional stretch $\lambda$, and the deformed radius $\bar{r}$ are known, the fundamental forms on $\overline{\mathscr{R}}$ are known and, from (6) and (7),

$$
\overline{\mathbf{r}}=\bar{r}(\sigma) \mathbf{e}_{r}(\theta)+\left[\bar{z}(0)+\int_{0}^{\sigma} \lambda(s) \sin \bar{\alpha}(s) d s\right] \mathbf{e}_{z} .
$$


Compatibility. By hypothesis, $\bar{r}(\sigma)$ and $\bar{z}(\sigma)$ have piecewise continuous second derivatives on $(0, L)$. Thus the vector compatibility condition

$$
\left(\overline{\mathbf{r}},{ }_{\theta}\right),{ }_{\sigma}=\left(\overline{\mathbf{r}},{ }_{\sigma}\right),{ }_{\theta}
$$

holds everywhere on $\mathscr{R}$ except, possibly, on a finite number of parallels. Inserting (6), (7), and the differential relation $\mathbf{e}_{r}^{\prime}(\theta)=\mathbf{e}_{z} \times \mathbf{e}_{r}(\theta)$ into (12), I obtain the scalar compatibility condition

$$
\vec{r}=\lambda \cos \bar{\alpha} \text {. }
$$

This is equivalent to Eq. (20) in [2]. Introducing meridional and hoop strains by setting

$$
\lambda=1+e_{\sigma} \text { and } \bar{r}=r\left(1+e_{\theta}\right),
$$

and recalling that $\lambda>0$, by assumption, I may rewrite (13) as

$$
\cos \bar{\alpha}=\frac{\left[r\left(1+e_{\theta}\right)\right]^{\prime}}{1+e_{\sigma}},
$$

which implies that

$$
\bar{\alpha}= \pm \operatorname{Cos}^{-1}\left\{\frac{\left[r\left(1+e_{\theta}\right)\right]^{\prime}}{1+e_{\sigma}}\right\} \pm 2 k \pi, \quad k=0,1,2, \ldots,
$$

where $\operatorname{Cos}^{-1}$ denotes the principal branch of the inverse cosine function. Theorem 1 is an immediate consequence of this expression and (10). Sign changes in (16) occur at points of inversion. The addition of multiples of $2 \pi$ in (16) can occur if a shell is folded over several times, as in Fig. 1, which shows a cylindrical shell $(\alpha=\pi / 2)$ of length $L$ that has been folded through an angle of $\pi$ at $\sigma=(1 / 2) L$ and through another angle of $\pi$ at $\sigma=(5 / 6) L$ so that its final configuration is not stretched. Thus, by (16), $\bar{\alpha}=(1 / 2) \pi$ if $0 \leq \sigma<(1 / 2) L, \bar{\alpha}=-(1 / 2) \pi+2 \pi$ if $(1 / 2)<\sigma<(5 / 6) L$, and $\bar{\alpha}=(1 / 2) \pi+2 \pi$ if $(5 / 6) L<\sigma \leq L$.

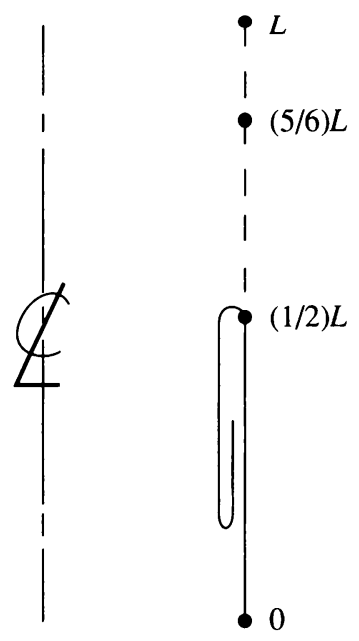

Fig. 1. Cylindrical shell folded on itself 
To establish Theorems 2 and 3, I set $r^{\prime}=\cos \alpha$ and use (14) and half-angle formulas to express (13) in the form

$$
\sin ^{2}(\bar{\alpha} / 2)=\sin ^{2}(\alpha / 2)+(1 / 2)\left[e_{\sigma} \cos \bar{\alpha}-\left(r e_{\theta}\right)^{\prime}\right] .
$$

To say that "the components of the strain and its first gradient are $O(\varepsilon)$ " means, in the present context, that $e_{\sigma}, e_{\theta}=O(\varepsilon)$ and that $\left(r e_{\theta}\right)^{\prime}=O(\varepsilon)$. Hence (17) implies that

$$
\sin (\bar{\alpha} / 2)= \pm \sqrt{\sin ^{2}(\alpha / 2)+O(\varepsilon)} .
$$

Moreover, $(14)_{2}$ yields

$$
\left|\frac{\bar{r}-r}{r}\right|=O(\varepsilon)
$$

which means that the relative radial deformation is $O(\varepsilon)$. Further, if I assume that the shell is translated axially so that $\bar{z}(0)=z(0)$, then (11) and $(14)_{1}$ imply that

$$
\left|\frac{\bar{z}-z}{L}\right|=\frac{1}{L} \int_{0}^{\sigma}|\sin \bar{\alpha}(s)-\sin \alpha(s)| d s+O(\varepsilon) .
$$

Theorems 2 and 3 now follow by noting that, at any fixed value of $\sigma$, two cases may be distinguished: (A) $\sin (\alpha / 2)=O\left(\varepsilon^{p}\right), 0 \leq p<1 / 2$, and $(\mathrm{B}) \sin (\alpha / 2)=O\left(\varepsilon^{1 / 2}\right)$.

In case A the shell is steep (nonshallow), and (18) implies that

$$
\sin (\bar{\alpha} / 2)= \pm \sin (\alpha / 2)+O\left(\varepsilon^{1-p}\right) .
$$

Thus,

$$
\bar{\alpha}= \pm \alpha \pm 2 k \pi+O\left(\varepsilon^{1-p}\right)
$$

so that $(20)$ implies

$$
\left|\frac{\bar{z}-z}{L}\right|=O\left(\varepsilon^{p}\right), \quad 0 \leq p<1 / 2 .
$$

Equations (19), (22), and (23) constitute Theorem 2, strengthened by the tighter bound of $O\left(\varepsilon^{p}\right)$ rather than $O(1)$ on the relative axial displacement.

In case B the shell is shallow, and (18) yields

$$
\sin (\bar{\alpha} / 2)=O\left(\varepsilon^{1 / 2}\right) .
$$

Thus,

$$
\bar{\alpha}= \pm 2 k \pi+O\left(\varepsilon^{1 / 2}\right)
$$

so that (20) implies

$$
\left|\frac{\bar{z}-z}{L}\right|=O\left(\varepsilon^{1 / 2}\right)
$$

Multiples of $2 \pi$ must be allowed in (25) to permit, for example, a deformation in which a narrow ring plate is turned inside out twice (and so returned to its initial configuration), as illustrated in Fig. 2. Equations (19), (25), and (26) constitute the modified version of Theorem 3 .

REMARK. The assumption that the strain gradient $\left(r e_{\theta}\right)^{\prime}$ is $O(\varepsilon)$ is overly restrictive and not required for classical nonlinear shell theory to hold. For example, in a sufficiently narrow ring plate turned inside out once, as in Fig. 2a, the strains remain 


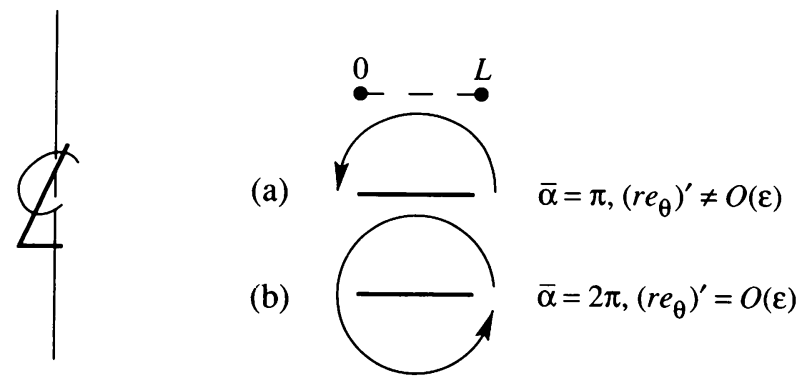

FIG. 2. A narrow ring plate turned inside out (a) once and (b) twice

small, even though the strain gradient may be $O(1)$. Less restrictive theorems might be obtained by removing the small bound on $\left(r e_{\theta}\right)^{\prime}$ but requiring that

$$
h\left|\bar{\alpha}^{\prime}-\alpha^{\prime}\right|=O(\varepsilon), \quad h\left|\frac{\sin \bar{\alpha}}{\bar{r}}-\frac{\sin \alpha}{r}\right|=O(\varepsilon),
$$

where $h$ is the thickness of the shell, that is, requiring that the strains at the outer fibers of the shell also be $O(\varepsilon)$.

\section{REFERENCES}

[1] P. M. Naghdi and L. Vongsarnpigoon, Some general results in the kinematics of axisymmetrical deformation of shells of revolution, Quart. Appl. Math. 43, 23-36 (1985)

[2] E. Reissner, On the theory of thin elastic shells, Contributions to Applied Mechanics (H. Reissner Anniversary Volume), J. W. Edwards, Ann Arbor, MI, 1949, pp. 231-247 